\title{
Servicios de salud en la Mixteca: utilización y condición de afiliación en hogares de migrantes y no-migrantes a EU
}

\author{
V Nelly Salgado de Snyder, PhD,(I) Tonatiuh González-Vázquez, Lic en C Pol,(I) César Infante-Xibille, PhD, (I) \\ Margarita Márquez-Serrano, M en Med Soc, (I) Blanca Pelcastre-Villafuerte, Dra en Psic Soc,(l) \\ Edson E Serván-Mori, M en Econ. ${ }^{(2)}$
}

\author{
Salgado de Snyder VN, González-Vázquez T, \\ Infante-Xibille C, Márquez-Serrano M, \\ Pelcastre-Villafuerte B, Serván-Mori EE. \\ Servicios de salud en la Mixteca: \\ utilización y condición de afiliación en hogares \\ de migrantes y no-migrantes a EU. \\ Salud Publica Mex 20 10;52:424-43 I.
}

\begin{abstract}
Resumen
Objetivo. Caracterizar a los hogares de la Mixteca baja en términos socioeconómicos y demográficos y analizar las diferencias entre miembros de hogares de migrantes (HogMig) y no migrantes (HogNoMig) a Estados Unidos en torno a su afiliación y utilización de servicios de salud. Material y métodos. Estudio transversal y descriptivo en el que se realizaron encuestas a jefes de familia de una muestra representativa de 702 hogares de la Mixteca baja con (HogMig) y sin miembros migrantes (HogNoMig) a EU. Resultados. Los integrantes de los HogMig tenían más recursos personales y económicos que los HogNoMig; además recibían remesas regularmente. La mayoría de los miembros de ambos tipos de hogares no recibía beneficios del Programa Oportunidades, ni contaba con afiliación al Seguro Popular, IMSS o ISSSTE. Generalmente utilizaban el centro de salud local, aunque frecuentemente preferían pagar médicos privados. La minoría derechohabiente (IMSS/ ISSSTE) reportó una muy baja utilización de esos servicios.
\end{abstract}

Palabras clave: migración internacional; servicios de salud; población rural; México
Salgado de SnyderVN, González-Vázquez T, Infante-Xibille C, Márquez-Serrano M,

Pelcastre-Villafuerte B, Serván-Mori EE.

Health services in the Mixteca:

use and conditions of affiliation

in US-migrant and non-migrant households. Salud Publica Mex 2010;52:424-43I.

\section{Abstract}

Objective. To describe the socioeconomic and demographic characteristics of households in the Mixteca Baja and analyze differences in affiliation with health care programs and utilization, among members of households with migrants (HogMig) and without migrants (HogNoMig) to the United States. Material and Methods. A cross-sectional, descriptive survey was used with heads of households in a representative sample from the Mixteca Baja of 702 homes with and without migrants to the US. Results. Members of HogMig had more personal and economic resources than those of HogNoMig; they also regularly received remittances. The majority of members of both HogMig and HogNoMig did not receive benefits from the Oportunidades program or health coverage through Seguro Popular, IMSS or ISSSTE. In general, while they used the local health clinic, they often preferred to pay for private practitioners. A small proportion of those covered by IMSS or ISSSTE reported very low utilization of the health services offered by those institutions.

Key words: emigration and immigration; health services; rural population; Mexico.

(I) Centro de Investigación en Sistemas de Salud. Instituto Nacional de Salud Pública. Cuernavaca, Morelos, México

(2) Centro de Investigación en Evaluación y Encuestas. Instituto Nacional de Salud Pública. Cuernavaca, Morelos, México

Fecha de recibido: I de septiembre de 2009 - Fecha de aceptado: 9 de junio de 2010 Solicitud de sobretiros:V. Nelly Salgado de Snyder. Instituto Nacional de Salud Pública. Av. Universidad 655, Col. Santa María Ahuacatitlán. 62100 Cuernavaca, Morelos, México.

Correo electrónico:nsnyder@insp.mx 
$E^{1}$ estado de salud es el resultado de las condiciones ociales en que la gente nace, crece, vive, trabaja y envejece, así como de los factores geográficos, culturales, económicos y sociales que determinan el acceso y utilización de los servicios de salud para prevenir y combatir las enfermedades. ${ }^{1}$ Bajo este esquema de determinantes sociales de la salud, las condiciones de vida de la población están directamente influenciadas por políticas sociales y económicas, tanto globales como del propio país. En particular, los problemas de salud de las personas están íntimamente vinculados con una distribución desigual del poder, ingresos, bienes y servicios, lo cual se refleja en una deficiente salud entre los más pobres.

La pobreza es el determinante social más importante en el deterioro de la salud. ${ }^{2}$ En México, país de ingresos medios, existen 44.7 millones de personas que viven en condiciones de pobreza de patrimonio y 14.4 millones en situación de pobreza alimentaria. ${ }^{3}$ Dos de cada tres personas con pobreza alimentaria viven en zonas rurales, en donde la pobreza es más acentuada que en las zonas urbanas y la escasez de recursos institucionales es la norma. ${ }^{4,5}$

En este contexto de desigualdad social se presenta la emigración hacia Estados Unidos (EU) como una alternativa para acceder a mejores condiciones de vida. Millones de mexicanos han emigrado a aquel país, frecuentemente ocupándose en trabajos mal remunerados y de altos riesgos para su salud como en la agricultura, construcción, manufactura y servicios. La migración indocumentada generalmente ocurre al margen de la economía formal y tiene implicaciones políticas, económicas, sociales y de salud para ambos países. ${ }^{6}$

La salud de los migrantes indocumentados y de sus familias que permanecen en México es uno de los retos más importantes para los sistemas de salud, ya que al no laborar en su país y no contar con permisos laborales en EU, los migrantes tienen menor derechohabiencia en México y limitado acceso a los servicios de salud en EU. Esta desprotección social en salud que enfrentan los migrantes mexicanos ha sido objeto de preocupación para ambos países desde hace décadas. ${ }^{7}$

Datos de 2007 sugieren que de los 11.8 millones de mexicanos que viven en EU, 56\% (6.7 millones) no contaba con algún tipo de cobertura de salud para atender sus enfermedades. ${ }^{8}$ Los problemas de salud reportados con mayor frecuencia entre los migrantes se han encontrado relacionados con estilos de vida cambiantes, demandas laborales excesivas y limitado acceso a servicios de salud. ${ }^{9,10}$ Problemas como diabetes, hipertensión, depresión y lesiones, que entre los migrantes son de prevalencia elevada, ${ }^{11}$ requieren de tratamientos especializados y de estrecho seguimiento médico, pero al no utilizar regularmente estos servicios, difícilmente son atendidos con éxito.

Una de las regiones de México con alta tradición migratoria a EU que en la actualidad sigue aportando mano de obra, mayoritariamente masculina, es la denominada Mixteca baja, localizada en la intersección de los estados de Puebla, Guerrero y Oaxaca. ${ }^{12}$ En esta región, las actividades laborales mal remuneradas en el sistema agrícola tradicional, junto con la exclusión social y la falta de oportunidades educativas, laborales, económicas y de desarrollo personal, ${ }^{13,14}$ han propiciado la emigración de sus pobladores para trabajar como jornaleros en el corte de caña en Veracruz y de jitomate en los estados de Morelos y Sinaloa. Muchos también migran a EU en búsqueda de una mejor calidad de vida y bienestar tanto individual como familiar. ${ }^{15-17}$ La Mixteca baja es una zona rural extremadamente árida, con baja producción agrícola y un alto grado de marginación que se ve reflejado en la gran proporción de viviendas con piso de tierra, sin drenaje y sin agua entubada ni servicios sanitarios. Sus pobladores se caracterizan por tener acceso muy limitado a bienes y servicios públicos, con altos niveles de analfabetismo -comparados con la tasa nacional- e ingresos menores al salario mínimo. ${ }^{18}$

El presente estudio tuvo como objetivo caracterizar a los hogares de la Mixteca baja en términos socioeconómicos y demográficos, así como analizar las diferencias entre hogares de migrantes y no migrantes en torno a la utilización de servicios de salud.

\section{Material y métodos}

Esta contribución se deriva de un proyecto de investigación que se llevó a cabo en el año 2005 en la región mexicana conocida como la Mixteca baja, en tres municipios rurales de los estados de Guerrero, Oaxaca y Puebla, clasificados por el Consejo Nacional de Población (Conapo) ${ }^{19,20}$ como de "alta" marginación y "muy alta" intensidad migratoria. Los municipios en los que se recolectó la información fueron Tlalixtaquilla de Maldonado, Guerrero; Santo Domingo Tonalá, Oaxaca; y Guadalupe Santana, Puebla.

Con el propósito de establecer un criterio de estratificación de los hogares, se definió un índice de nivel socioeconómico (NSE). ${ }^{21}$ Dicho índice se construyó a través de la matriz de correlación policórica de un análisis de componentes principales ${ }^{22}$ y conjuga información sobre las condiciones de infraestructura y posesión de activos en el hogar (variables dicotómicas; sí = 1, no = 0 ): materiales utilizados para techos, paredes y pisos (durables o no), la existencia o no de servicios de agua entubada y electricidad, así como posesión de estufa, refrigerador, televisores, equipos de sonido, teléfono, 
lavadora, horno de microondas y computadoras. A partir del indicador de nivel socioeconómico se generaron terciles de distribución de la muestra de análisis, lo cual permitió establecer un criterio de estratificación de la condición económica de los hogares.

Para la descripción de la muestra, se calcularon medidas de tendencia central y de dispersión, para las variables continuas, y de frecuencias y porcentajes, para las variables categóricas, y se realizaron además pruebas de diferencias marginales (al 1, 5 y 10\%) de Wald en medias y proporciones de los principales indicadores analizados.

\section{Diseño muestral}

El diseño muestral fue parte de un proceso de muestreo por clusters al interior de los tres municipios arriba mencionados, multietápico y estratificado por localidades, ${ }^{23}$ para el que se construyó un índice de selección aleatoria ponderado por número de hogares, de tal manera que aquellas localidades con mayor número de hogares tuvieran mayor probabilidad de ser seleccionados. Este procedimiento permitió definir un total de 19 localidades en donde se realizó el trabajo de campo (seis en Tlalixtaquilla, seis en Tonalá y siete en Guadalupe), que incluyeron un total de 702 hogares con representatividad regional. En cada unidad doméstica, previa firma de consentimiento informado, se entrevistó al jefe de hogar $(\mathrm{JH})$ y a otros integrantes de la unidad. En este trabajo se reportan únicamente los datos recabados en las encuestas a los JH, quienes proporcionaron información acerca de los 3841 miembros de las 702 unidades domésticas estudiadas.

Adicionalmente, se realizaron 42 entrevistas semiestructuradas a mujeres encargadas del cuidado de los miembros de las unidades domésticas para explorar varios aspectos del proceso de atención a la salud; aquí sólo reportaremos su experiencia sobre la utilización de servicios de salud. Esta información cualitativa permitió generar líneas explicativas para los resultados numéricos, y es en este sentido que se incorporan en la sección de Discusión del presente trabajo, sin pretensión de ser concluyentes.

Finalmente, las encuestas y entrevistas fueron administradas por personal expresamente capacitado para este propósito. El proyecto fue a probado por la Comisión de Ética del Instituto Nacional de Salud Pública.

\section{Definición de los hogares migrantes}

Los 702 hogares de la muestra fueron caracterizados como uno de dos posibles tipos de hogares: Hogar no migrante(HogNoMig), en el que el jefe de hogar reportó que ninguno de sus miembros había vivido o laborado en EU, y Hogar migrante (HogMig) en el que: 1) al menos un miembro de ese hogar trabajaba en EU (migrante activo), o 2) al menos un miembro de ese hogar había laborado en EU por lo menos durante un año, y al momento de la encuesta residía en la localidad en que se llevó a cabo el estudio (migrante de retorno). Del total de hogares, 39\% fueron HogNoMig y 61\% HogMig; de los cuales, $31 \%$ contaba con migrantes activos, $19 \%$ con migrantes de retorno y $11 \%$ tenía tanto migrantes activos como de retorno.

Las variables estudiadas en el proyecto de investigación fueron numerosas, pero para el presente trabajo se analizaron únicamente las siguientes: características sociodemográficas de los miembros de las unidades domésticas (edad, sexo, escolaridad, condición laboral), número de integrantes del hogar y características de la vivienda, derechohabiencia al Instituto Mexicano del Seguro Social (IMSS), Instituto de Seguridad y Servicios Sociales para los Trabajadores del Estado (ISSSTE), afiliación al Seguro Popular, utilización de servicios de salud y si recibían o no apoyo de programas gubernamentales.

\section{Resultados}

El cuadro I describe las principales características de los miembros del hogar y su vivienda, así como las diferencias encontradas entre HogNoMig y HogMig.

En relación a sus integrantes, en todos los hogares se presentaron proporciones semejantes de hombres y mujeres. Los HogMig se caracterizaron por tener, en promedio, integrantes más jóvenes, que hablaban español y contaban con más años de escolaridad (un año más aproximadamente) que sus contrapartes en $\mathrm{HogNoMi}$ gG $(p<0.01)$. Por su parte, en los HogNoMig se observó que sólo 30\% $(p<0.01)$ de sus miembros contaba con trabajo remunerado (7.5\% menos que en los HogMig), un mayor porcentaje eran mixteco-hablantes $(p<0.01)$ y analfabetos (alrededor de 3\% más, $p<0.01$ ), en relación con los HogMig.

Los JH en HogMig, comparados con los HogNoMig, eran en su mayoría hombres, más jóvenes (6 años menos), con aproximadamente un año más de escolaridad y hablaban menos el mixteco; pese a esto no se encontraron diferencias en su incorporación al mercado laboral (alrededor de 64\% en ambos tipos de hogares). En cuanto a las características de la vivienda, los HogMig contaban en su mayoría con viviendas propias y tenían un mayor número de habitaciones e integrantes en la unidad doméstica que los HogNoMig $(p<0.01)$.

En dicho cuadro se aprecia también que un mayor porcentaje de HogMig, en relación con los HogNoMig, 
Cuadro I

Características sociodemográficas de los PARTICIPANTES POR tipo de hogar No MigRante y MigRante en la Mixteca. MéXico, 2005

\begin{tabular}{|c|c|c|c|c|c|c|c|}
\hline \multirow{2}{*}{ Características } & \multicolumn{2}{|c|}{ Total } & \multicolumn{2}{|c|}{ HogNoMig } & \multicolumn{2}{|c|}{ HogMig } & \multirow{2}{*}{$\begin{array}{c}\text { Valor } \\
p\end{array}$} \\
\hline & $N$ & \% o Media & $N$ & Media & $N$ & Media & \\
\hline \multicolumn{8}{|l|}{ De los integrantes del hogar } \\
\hline Edad en años & 3841 & 29.7 & 1502 & 32.3 & 2339 & 27.9 & 0.00 \\
\hline \multicolumn{8}{|l|}{ Sexo } \\
\hline Hombres & 3841 & 48.7 & 1502 & 47.1 & 2339 & 49.6 & 0.13 \\
\hline Mujeres & 3841 & 51.3 & 1502 & 52.9 & 2339 & 50.4 & 0.13 \\
\hline Trabaja & 3710 & 34.4 & $147 \mid$ & 29.9 & 2239 & 37.4 & 0.00 \\
\hline Escolaridad en años & 3669 & 4.5 & 1436 & 4.1 & 2233 & 4.8 & 0.00 \\
\hline Sin educación & 3669 & 24.1 & 1436 & 28.1 & 2233 & 21.6 & 0.00 \\
\hline Alfabeta & 3722 & 74.7 & 1475 & 70.7 & 2247 & 77.4 & 0.00 \\
\hline Habla español & 3751 & 97.4 & 1472 & 95.9 & 2279 & 98.3 & 0.00 \\
\hline Habla mixteco & 3751 & 17.9 & 1472 & 20.2 & 2279 & 16.3 & 0.00 \\
\hline \multicolumn{8}{|l|}{ Del jefe de hogar } \\
\hline Edad en años & 696 & 50.8 & 326 & 54.7 & 370 & 47.4 & 0.00 \\
\hline \multicolumn{8}{|l|}{ Sexo } \\
\hline Hombres & 693 & 61.6 & 325 & 58.5 & 368 & 64.4 & 0.11 \\
\hline Mujeres & 693 & 38.4 & 325 & 41.5 & 368 & 35.6 & 0.11 \\
\hline Trabaja & 694 & 63.8 & 326 & 64.4 & 368 & 63.3 & 0.76 \\
\hline Escolaridad en años & 683 & 3.5 & 316 & 3.1 & 367 & 3.8 & 0.01 \\
\hline Sin educación & 683 & 31.3 & 316 & 37.3 & 367 & 26.2 & 0.00 \\
\hline Alfabeta & 691 & 69.6 & 325 & 65.2 & 366 & 73.5 & 0.02 \\
\hline Habla español & 695 & 98.3 & 326 & 98.2 & 369 & 98.4 & 0.84 \\
\hline Habla mixteco & 695 & 17.4 & 326 & 17.5 & 369 & 17.0 & 0.95 \\
\hline \multicolumn{8}{|l|}{ Del hogar } \\
\hline Número de integrantes & 697 & 4.7 & 327 & 4.4 & 370 & 4.9 & 0.01 \\
\hline Número de cuartos & 692 & 3.5 & 326 & 3.2 & 366 & 3.8 & 0.00 \\
\hline Vivienda propia & 696 & 84.6 & 327 & 82.9 & 369 & 86.2 & 0.00 \\
\hline NSE bajo & 698 & 32.5 & 328 & 39.9 & 370 & 25.9 & 0.00 \\
\hline NSE medio & 698 & 32.5 & 328 & 32.9 & 370 & 32.2 & 0.84 \\
\hline NSE alto & 698 & 32.4 & 328 & 25.6 & 370 & 38.4 & 0.00 \\
\hline
\end{tabular}

HogMig= hogares de migrantes

HogNoMig= hogares de no migrantes

Fuente: Elaboración propia con datos del proyecto "Migración, salud y salud mental en México rural en el contexto de la globalización” 2005

se ubicaron en el NSE alto (14\% más, $p<0.01)$, mientras que la mayoría de los HogNoMig se concentraron en el NSE bajo (13\% más, $p<0.01)$. Alrededor de una tercera parte de ambos grupos de hogares se colocaron en el NSE medio. Aunque no se muestra en el cuadro I, cabe mencionar que las viviendas de los
HogMig poseían en promedio mejores condiciones de infraestructura (en piso, techo y paredes) y más activos como refrigerador, estufa, televisor, etc. que los HogNoMig.

En los HogMig se identificaron un total de 646 miembros migrantes activos o de retorno. En estos 
hogares, los jefes de familia reportaron que la mayoría $(86.4 \%)$ de sus miembros migrantes no contaba con documentos legales para residir y trabajar en EU y que $85.8 \%$ mandaba remesas regularmente. ${ }^{*}$

Con el fin de brindar una idea de los problemas que enfrentan diferencialmente los miembros de HogMig y HogNoMig en esta zona geográfica, el cuadro II proporciona un acercamiento general a algunos indicadores del estado de salud de dos grupos vulnerables: mujeres en edad reproductiva y ancianos

\footnotetext{
* La razón fundamental para emigrar fue búsqueda de trabajo con el propósito de mejorar su economía y mantener a la familia, pagar deudas, etc. (91.4\%). El resto (8.6\%) emigró a EU por otras razones (curiosidad, estudiar, conocer). Las remesas eran utilizadas en el hogar por orden de importancia en los siguientes rubros: alimentos, compra de bienes muebles e inmuebles, compra de ropa y calzado, pago de servicios y gastos médicos.
}

(hombres y mujeres). Los padecimientos mostrados fueron diagnosticados por un profesional de la salud en el último año y reportados por los afectados. Las mujeres de HogMig reportaron más enfermedades de transmisión sexual $(p>0.15)$, enfermedades crónicas como hipertensión y diabetes $(p>0.1)$ y, aunque no de manera significativa, más enfermedades infecciosas como tuberculosis y neumonía. Por su parte, entre los ancianos, aunque no se encontraron diferencias estadísticas, aquellos ubicados en HogNoMig reportaron una mayor presencia de enfermedades de los huesos, crónicas, infecciosas y de visión.

El cuadro III presenta datos de afiliación al Programa Oportunidades, Seguro Popular y derechohabiencia a instituciones de salud. De los 3841 miembros de todos los hogares, sólo 716 (18.6\% del total de la muestra) eran beneficiarios de Oportunidades, con una proporción similar de HogMig y HogNoMig afiliados

\section{Cuadro II}

\section{Problemas de salud en mujeres en edad productiva y ancianos de ambos sexos por tipo de hogar no migrante y migrante en la MiXteCA. México, 2005}

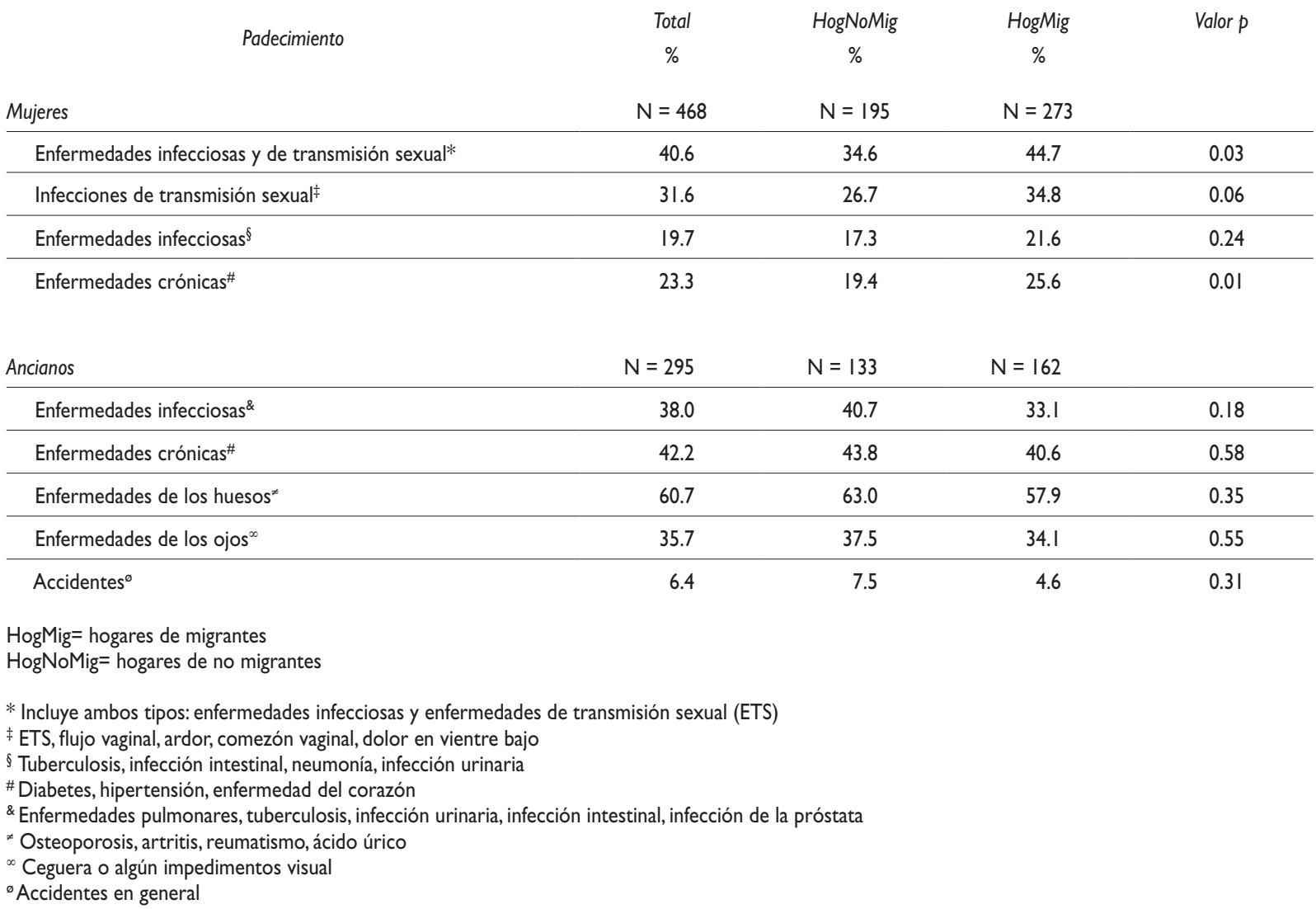

Fuente: Elaboración propia con datos del proyecto “Migración, salud y salud mental en México rural en el contexto de la globalización” 2005 
Cuadro III

Participación en programas sociales, afiliación para recibir atención a la salud y utilización de Servicios por tipo de hogar no migrante y migrante en la MixteCA. México, 2005

\begin{tabular}{|c|c|c|c|c|c|c|c|}
\hline \multirow{2}{*}{ Características } & \multicolumn{2}{|c|}{ Total } & \multicolumn{2}{|c|}{ HogNoMig } & \multicolumn{2}{|c|}{ HogMig } & \multirow{2}{*}{ valor $p$} \\
\hline & $N$ & $\%$ o Media & $N$ & \% o Media & $N$ & \% o Media & \\
\hline Beneficiario de algún programa social' & 3487 & 23.7 & 1455 & 20.8 & 2032 & 23.7 & 0.94 \\
\hline Beneficiario de Oportunidades & 3846 & 18.6 & 1509 & 19.4 & 2337 & 18.0 & 0.28 \\
\hline \multicolumn{8}{|l|}{ Afiliación para recibir servicios médicos } \\
\hline IMSS & 3872 & 5.6 & 1516 & 5.9 & 2356 & 5.5 & 0.60 \\
\hline ISSSTE & 3872 & 1.9 & 1516 & 3.0 & 2356 & 1.3 & 0.00 \\
\hline Seguro Popular & 3872 & 13.6 & 1516 & 15.3 & 2356 & 12.6 & 0.02 \\
\hline \multicolumn{8}{|c|}{ Centro de salud al que acude a atenderse regularmente } \\
\hline De la comunidad & 3394 & 57.3 & 1469 & 59.8 & 1925 & 55.4 & 0.01 \\
\hline Privado & 3394 & 22.3 & 1469 & 19.7 & 1925 & 25.7 & 0.00 \\
\hline Privado y de la comunidad & 3394 & 10.1 & 1469 & 11.6 & 1925 & 8.9 & 0.01 \\
\hline IMSS/ISSSTE & 3394 & 5.5 & 1469 & 5.3 & 1925 & 5.6 & 0.70 \\
\hline
\end{tabular}

* Incluye Oportunidades, Procampo, Piso Firme, Tu Casa, y otros apoyos de entidades federales [Desarrollo Integral de la Familia (DIF)], estatales (Pensión Guerrero) y de la sociedad civil (Niños de México)

IMSS: Instituto Mexicano del Seguro Social

ISSSTE: Instituto de Seguridad y Servicios Sociales de los Trabajadores del Estado

Fuente: Elaboración propia con datos del proyecto “Migración, salud y salud mental en México rural en el contexto de la globalización” 2005

al mismo. En cuanto a derechohabiencia, 78\% de todos los hogares reportó no tener miembros afiliados a instituciones de salud. $\mathrm{Al}$ analizar los datos por condición de aseguramiento, encontramos que una proporción significativamente mayor de miembros de HogNoMig que de HogMig estaban afiliados al ISSSTE $(p<.0 .01)$ y al Seguro Popular $(p<0.05)$. No se encontraron diferencias en cuanto a derechohabiencia al IMSS, con un porcentaje reducido de afiliación en ambos tipos de hogares.

La parte inferior del cuadro III presenta el lugar a donde acudieron regularmente los miembros de los hogares entrevistados cuando requerían servicios de salud. Como se observa, un porcentaje significativamente mayor $(p<0.01)$ de miembros de HogMig $(25.7 \%)$ que de HogNoMig (19.7\%) acudía a servicios privados de cuidados a la salud. Los HogNoMig utilizaban más los servicios que se ofrecían, tanto en la comunidad a través de los centros de salud locales $(p<0.05)$, como en una combinación de servicios públicos y privados $(p<0.05)$. Finalmente, no se detectaron diferencias significativas entre ambos grupos en el uso regular de servicios de salud ofrecidos por el IMSS o ISSSTE; un porcentaje menor a 6\% de los miembros de HogMig y HogNoMig acudían regularmente a las instituciones de salud de las cuales eran derechohabientes.

\section{Discusión}

Como se ha mencionado en esta contribución, la Mixteca baja es una región que se caracteriza por tener un elevado nivel de marginación y un alto índice de intensidad migratoria hacia los EU. En este contexto, la migración hacia ese país para muchas familias se ha convertido en una opción de sobrevivencia y bienestar, dadas las escasas oportunidades que existen en la región para el desarrollo social, económico y personal de sus habitantes. En esta región se observa un estado homogéneo de pobreza generalizada, sin embargo, los hallazgos de este estudio sugieren la existencia de diferencias en características socioeconómicas y demográficas entre los pobladores de la región, concretamente entre hogares que reportan tener al menos un integrante que es migrante (activo o de retorno) a los EU (HogMig) y los hogares que no tienen migrantes (HognoMig). También se observaron diferencias en la utilización de servicios de salud entre los HogMig y los HogNoMig.

Los resultados de este trabajo sugieren que los HogMig cuentan con mayores recursos personales y económicos que les permiten tener mayor acceso a bienes y servicios, lo cual se traduce en un nivel socioeconómico más alto en comparación con los miem- 
bros de los HogNoMig. Asimismo, los HogMig tienen un mayor número de integrantes, son más jóvenes y cuentan con mayor escolaridad en comparación con los HogNoMig.

Al ser la Mixteca baja una zona de alta marginación, llama la atención que menos de una quinta parte de la muestra total de hogares recibía los beneficios de Oportunidades, programa que busca fomentar el desarrollo humano de la población en pobreza extrema a través de apoyos en educación, salud, nutrición e ingreso. Una proporción aún menor reportó afiliación al Seguro Popular; no obstante, al ser entrevistadas algunas mujeres señalaron que unas semanas antes de esta encuesta se habían inscrito al Seguro Popular y aún no habían utilizado los servicios que ofrecía ese programa. Sin embargo, sus expectativas no eran positivas, pues estaban basadas en experiencias previas en el centro de salud local y otros servicios de salud y pensaban que el Seguro Popular no respondería adecuadamente a sus necesidades. Lo anterior señala un importante problema de actitud que podría transformarse en conductas de no utilización de estos servicios.

En general existe baja afiliación a los dos sistemas más importantes de seguridad social en México: el IMSS y el ISSSTE. Asimismo, identificamos diferencias entre los tipos de hogares, con una proporción significativamente mayor de miembros de HogNoMig afiliados al ISSSTE y al Seguro Popular.

La utilización de servicios privados de salud fue mayor en los HogMig, mientras que los HogNoMig utilizaban más los servicios ofrecidos por los centros de salud locales. A pesar de que $57 \%$ de los miembros de ambos hogares utilizaba servicios de salud públicos, las mujeres entrevistadas percibían que éstos eran de baja calidad y manifestaron que preferían acudir a servicios privados. Percibían también que la mayoría de las personas que contaban con derechohabiencia en el IMSS o ISSSTE no utilizaban estos servicios debido al gasto y tiempo que implicaba transportarse a las comunidades en donde se localizaban estas instituciones y acudían entonces a los centros de salud locales o a médicos privados. Esta percepción de baja calidad podría explicar por qué, cuando tienen la posibilidad, prefieren pagar servicios de salud privados; además, denota insatisfacción con un servicio al que acuden por ser la única opción, más que por convicción.

Esta situación da cuenta de una problemática sumamente compleja que se encuentra relacionada con el limitado uso de servicios de salud del IMSS e ISSSTE y con la deficiente calidad de los servicios que prestan los centros de salud dirigidos a población abierta. La atención en salud, desde la perspectiva de los determinantes sociales de la salud, es un determinante intermedio en los resultados, que a su vez se vinculan con la respuesta del sistema a las necesidades de la población.

La aparente predisposición a pagar por servicios de salud privados nos orienta sobre el riesgo al que están expuestos particularmente aquellos grupos que viven en contextos de pobreza generalizada, como la región de la Mixteca baja, en donde la inequidad se traduce en mayor gasto de bolsillo para cubrir las necesidades de atención en salud.

De acuerdo con el reporte de la Comisión de Determinantes Sociales de la Salud de la OMS, ${ }^{1}$ las políticas actuales en muchos países se basan casi exclusivamente en modelos de crecimiento urbano, olvidando las necesidades de infraestructura y servicios en zonas rurales. Esto tiende a desencadenar y promover la emigración hacia centros urbanos nacionales y hacia otros países. En el caso de México, la emigración hacia EU en zonas marginadas como la Mixteca baja se ha institucionalizado y representa una opción trascendental de sobrevivencia para sus pobladores.

El reto que claramente puede ser identificado en este escenario son las barreras culturales (incluidas las relacionadas con el lenguaje), sociales y económicas que enfrentan los pobladores de la Mixteca baja para la utilización de servicios de salud. Así, la pregunta central debe ser planteada en torno al cómo diseñar, abordar, llevar, promover y evaluar servicios de salud de calidad a los pobladores de las localidades más remotas y empobrecidas. En este ámbito, resulta esencial continuar el trabajo iniciado en la tercera generación de reformas al sistema nacional de salud que tiene como propósito la implementación de una cobertura universal en salud que incorpore equidad, calidad y protección financiera. ${ }^{24}$

Es evidente que estas metas tienen implicaciones que van más allá de la salud; son temas de justicia social que deben ser atendidos de manera prioritaria, estratégica, sostenida y coordinada junto con otros sectores. Es necesario que los desafíos que actualmente enfrenta el sistema de salud en torno a la migración y la pobreza sean no únicamente identificados, sino investigados a profundidad para determinar su magnitud y actuar en consecuencia.

Ésta, como todas las investigaciones sociales, tiene limitaciones. Una de ellas es que este estudio, al ser transversal, no nos permitió identificar si los mayores recursos personales y materiales en los HogMig ya existían y fueron los factores que de alguna manera promovieron la migración de sus miembros, o si el mejoramiento de sus condiciones personales y materiales ocurrió como consecuencia de la migración. Esta última noción parecería ser la respuesta más adecuada, ya que la razón fundamental para emigrar a $\mathrm{EU}$, de acuerdo con 
la mayoría de los participantes en el estudio, fue mejorar la economía familiar. Otra limitación del diseño fue que no permitió medir el impacto gradual de las remesas recibidas en los HogMig sobre la utilización de servicios de salud. Finalmente, es posible que estos resultados reflejen un sesgo en la respuesta de los participantes, ya que nuestro levantamiento de datos coincidió con el periodo de tiempo en que se llevó a cabo el proceso de afiliación al Seguro Popular en la región.

\section{Agradecimientos}

El presente trabajo se realizó con recursos de The Wellcome Trust, Reino Unido. Grant GR074006MA, a través del proyecto Migration, health and mental health in rural Mexico in the context of globalization. Agradecemos al equipo de trabajo que participó en este proyecto (encuestadores, supervisores, operadores, apoyo secretarial y capturistas) y a la coordinación general y los operadores estatales del Programa Vete Sano Regresa Sano, de la Dirección General de Promoción de la Salud de la Secretaría de Salud de México.

\section{Declaración de conflicto de intereses}

Declaramos no tener conflicto de intereses.

\section{Referencias}

I. Marmot M. Achieving health equity: from root causes to fair outcomes. The Lancet 2007;370: I 153-1 I63.

2. Organización Mundial de la Salud. Closing the gap in a generation. Final Report of the Commission on Social Determinants of Health. Ginebra: OMS, 2008.

3. Encuesta Nacional de Ingresos y Gastos de los Hogares. México, DF: INEGI, 2006.

4. Consejo Nacional de Evaluación de la Política de Desarrollo Social. Medición de la Pobreza [Online] [Consultado el 20 de abril de 2009]. Disponible en: http://www.coneval.gob.mx/coneval2/htmls/medicion_ pobreza/HomeMedicionPobreza.jsp?categorias=MED_POBREZA,MED_ POBREZA-med_pob_ingre

5. Morales-Ramos E. La evaluación de la pobreza difusa multidimensional en México, 1994-2006. México, DF: Banco de México, 2009.

6. PNUD, Informe sobre desarrollo humano, México 2006-2007. México, DF: Mundiprensa, 2007.

7. González-Block MA, Becker-Dreps S, De la Sierra-de la Vega LA, York P, Gardner S, González LM, et al. Salud Migrante. Propuesta de un seguro binacional. Perspectivas en Salud Pública. Cuernavaca Morelos, México: Instituto Nacional de Salud Pública, 2008.

8. CONAPO. Migración y salud: latinos a los Estados Unidos. México, DF: CONAPO, 2008.

9. Asakura $\mathrm{H}$. Experiencias en espiral. Remesas sociales y servicios de salud en una comunidad mixteca. En: Suárez B, Zapata E, coord. llusiones, sacrificios y resultado: El escenario real de las remesas de emigrantes a Estados Unidos. México DF: GIMTRAP, 2007: 59-103.

10. Bade B. Yerbas, limpias y cirugía: el cuidado de la salud entre los mixtecos de California. En: Escárcega S,Varese S, coord. La ruta mixteca. México DF: UNAM, 2004; 27I-3I4.

II. Salgado-de Snyder VN, González T, Bojórquez I, Infante C. Migración México-Estados Unidos: Consecuencias para la salud. Perspectivas en Salud Pública. Cuernavaca, Morelos, México: Instituto Nacional de Salud Pública, 2007

12. Sylvia E,Varese S. La ruta mixteca. México: UNAM, 2004.

13. Ibarra-Mateos M. De Nueva York a la Mixteca:Algunas consideraciones en torno al papel de las remesas en la economía nacional, regional y local. Memoria del Seminario Internacional sobre la Transferencia y el uso de las Remesas: Proyectos Productivos y de Ahorro. Zacatecas, Zac. 3-5 de octubre 200I.

14. Tlachinollan. Migrar o morir, Jornaleros agrícolas en los campos tóxicos de Sinaloa (Video documental). México: Promedios / Tlachinollan. 2008

15. Resano E, Wong R, Martínez L. El ingreso en hogares con migración hacia Estados Unidos. Papeles de Población, No. 39, Enero-marzo, 2004: 37-55.

16. Salgado de Snyder VN. Motivaciones de la migración de mexicanos hacia los Estados Unidos. En: MM de Alba Medrano, ed. Temas selectos de salud y derecho. México: UNAM, Instituto de Investigaciones Jurídicas, 2002;89-108.

17. Escárcega S,Varese S, coord. La ruta mixteca. México DF: UNAM, 2004: 27|-3|4.

18. Consejo Nacional de Población. Índices de marginación, 2005. México, DF: CONAPO, 2006.

19. Consejo Nacional de Población. Índices de intensidad migratoria México-Estados Unidos, 2000. México, D.F.: CONAPO 2002. [Online]'.

Consultado el 7 de octubre de 2008. Disponible en: http://www.conapo. gob.mx/publicaciones/migra4.htm

120. Consejo Nacional de Población. Índices de Marginación 2000. México, DF: CONAPO (200I). [Consultado el 7 de octubre de 2008]. Disponible en: http://www.conapo.gob.mx/publicaciones/indice2000.htm. 2I. McKenzie D. Measuring inequality with assets indicators. Journal of Population Economics 2005; 18(2): 229-260.

22. Kolenikov $\mathrm{S}$, Angeles $\mathrm{G}$. The use of discrete data in principal component analysis: theory, simulations, and applications to socioeconomic indices. Working Paper of MEASURE/Evaluation project, No.WP-04-85, Carolina Population Center, UNC. 2004.

23. Bennett S, Woods T, Liyanage WM, Smith DL.A simplified general method for cluster-sample surveys of health in developing countries. World Health Statitics Quaterly 1991; 44(3):98-106.

24. Frenk J, Sepúlveda J, Gómez-Dantés, Knaul F. Evidence-based health policy: three generations of reform in Mexico. The Lancet 2003; 362: |667-|67|. 